\title{
Visual estimation of the force applied by another person
}

${ }_{3}$ Yusuke Ujitoko ${ }^{1, *}$ and Takahiro Kawabe ${ }^{1}$

\author{
${ }^{1}$ NTT Communication Science Laboratories, Nippon Telegraph and Telephone Corporation, Atsugi, 243-0198, \\ Japan \\ *yusuke.ujitoko@gmail.com
}

\section{ABSTRACT}

As observers, we believe that we can visually estimate the force that another person applies to an object. However, it is unclear what kind of cues we use to do this. We have focused on two types of visual change that occur when a person pushes an elastic object from above with his or her finger: the first one relates to a finger/hand shaking, known as an "induced tremor" and the second one relates to object deformation due to the application of force. This study shows that human observers of videos combine these two visual cues to estimate the force being applied by another person in the videos. Overall, the apparent force was stronger when the shaking was larger and when the magnitude of the deformation was larger, although systematic individual differences existed. The estimation of force was likely made in separate cognitive functions from the estimation of object softness. Estimating the force that another person applies seems to be done by perceptually interpreting both the actions of others and their external outcomes.

By observing the motor actions of another person, we can infer her/his intention, anticipate the consequence of her/his action, and estimate the physical properties of an object that she/he is manipulating ${ }^{1-8}$. For example, professional basketball players can predict the results of another player' $s$ shot by visually reading the shooter's body kinematics ${ }^{1}$. Interestingly, in Anglioti's study ${ }^{1}$, the basketball players who was watching the video exhibited a time-selective motor activation, consistent with the previous evidence for the close relationship between seeing and conducting motor action in the brain ${ }^{9}$. Indeed, the combination of observation and imitation of another person's action has been found to be effective for a non-athlete group in learning new sports ${ }^{10}$. The ability of the observer to visually read the kinematics of another person's action is also useful for judging the intrinsic property of an object. Specifically, the kinematics of hand motion of another person is used as a cue to predict the size $\mathrm{e}^{5,6}$ and weight ${ }^{7,8}$ of an object that she/he is going to grasp.

However, the ability of human observers to visually determine what amount of force another person applies to an external object has not been examined in the literature. One of the bodily changes that often occur when a person applies force to an object is an induced tremor, which is a self-maintaining force fluctuation observed when one's muscles contract anisometrically under appropriate loading ${ }^{11}$. Depending on the strength of loading, the power peak of the induced tremor is seen at a temporal frequency of $4-8 \mathrm{~Hz}$. It is an intriguing question whether human observers can use the visual feature corresponding to the induced tremor to judge the amount of force that another person applies to an external object.

In addition to changes in another person' s physical behavior, the amount of change in the appearance of an external object is possibly a cue to the force being applied by that person. For example, the more force the player transfers to a ball when hitting the ball with a golf club, the farther the ball will fly. The stronger the force with which a cushion is pushed in, the larger the cushion's deformation. It has not been clarified whether the different amount of visual change in the appearance of an external object could contribute to the determination of the force applied.

Here we show that human observers can visually estimate the amount of force that another person applies by using the visual features of the induced tremor and the visual deformation of an external object. In the stimulus video clip, a scene in which an actor pressed a 3D printed elastic object (Fig. 1a) with his finger was presented. In order to mimic the induced tremor generally seen when a person pushes an elastic object with her/his finger, the actor slightly vibrated his hand and fingers while pushing the object. We call this vibration of his hand and fingers as imitated shaking. As shown in Results, the imitated shaking in his hand and fingers had a similar temporal frequency characteristics to that of an induced tremor. We hypothesized that observers would estimate larger forces being applied by the actor when they saw a greater shaking of the hand and fingers. We also used several elastic objects, each having a different force-displacement curve. As such, even if the actor pushed the objects with approximately equivalent forces, the magnitude of deformation (indentation) varied across the objects depending on their force-displacement curves (Fig. 1b). We hypothesized that observers would estimate larger forces when they watch larger deformations (or indentations) of the object. The results revealed that, in general, the presence of large imitated shaking, 
as well as a large deformation of the objects, increased the estimated force rating. However, further analysis showed that there were some observer groups in which the observers tended to use the shaking motion and deformation magnitudes in different ways from the major group of observers. We also observed that the difference in the usage of visual information among the observers was not evident for the estimation of softness of the elastic objects, which suggests that the estimation of applied force is made in a separate cognitive function from the softness estimation. The results indicate that the visual feature of the induced tremor and the apparent magnitude of object deformation are effective cues to the force that another person applies, while the usage of this visual information is dependent on what sort of assumptions the observers make for the cause of the tremor and object deformation. 
a

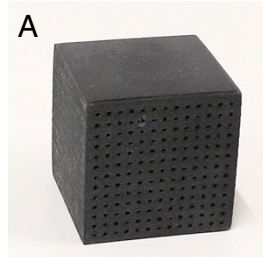

B

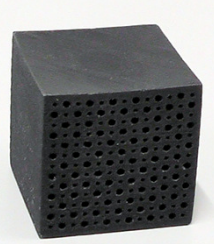

C

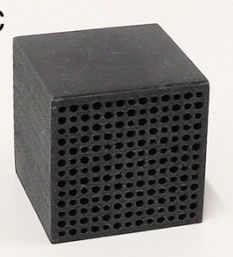

D

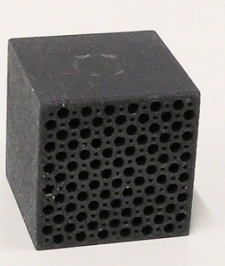

E

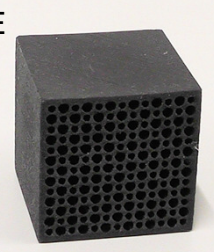

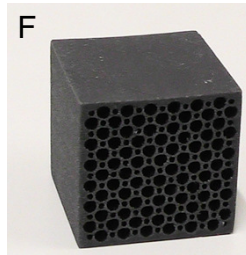

C
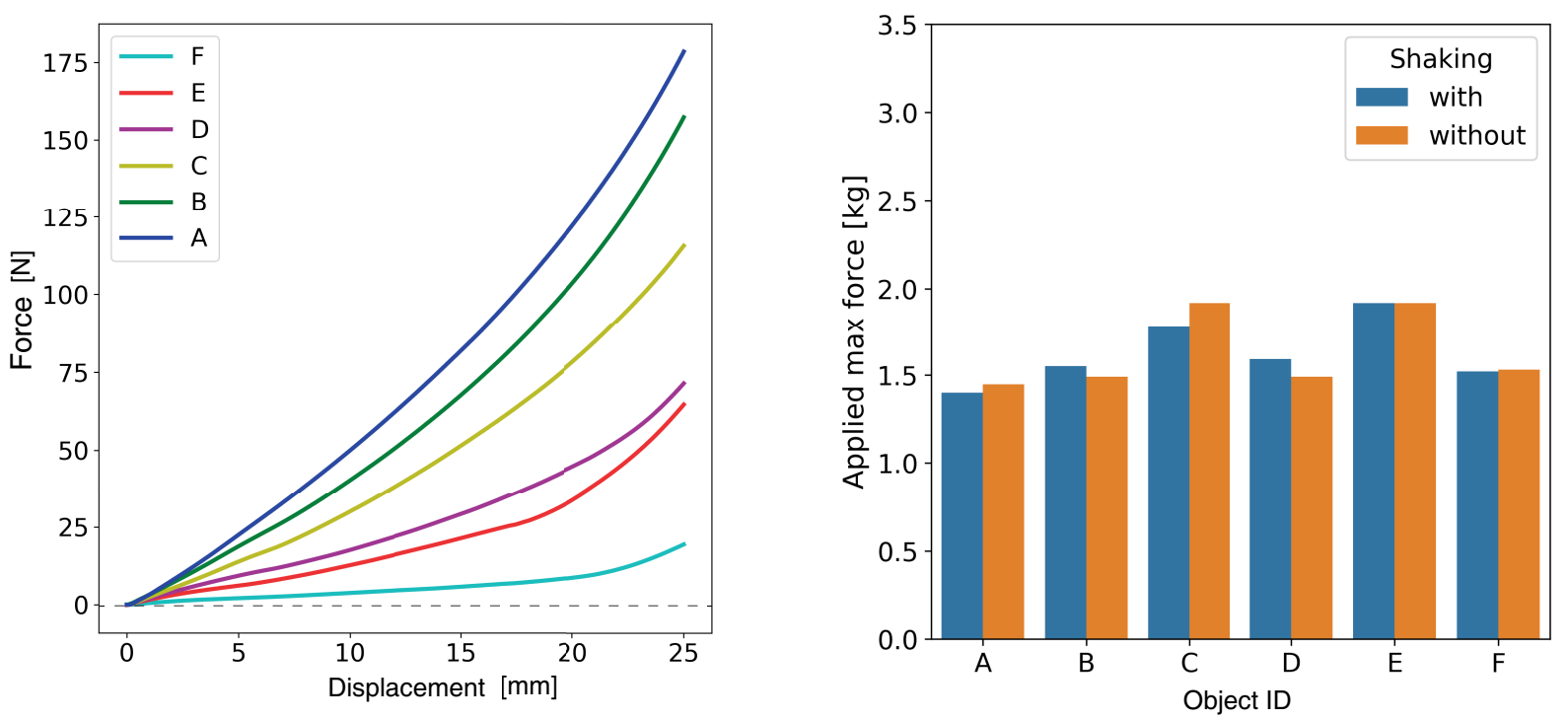

d

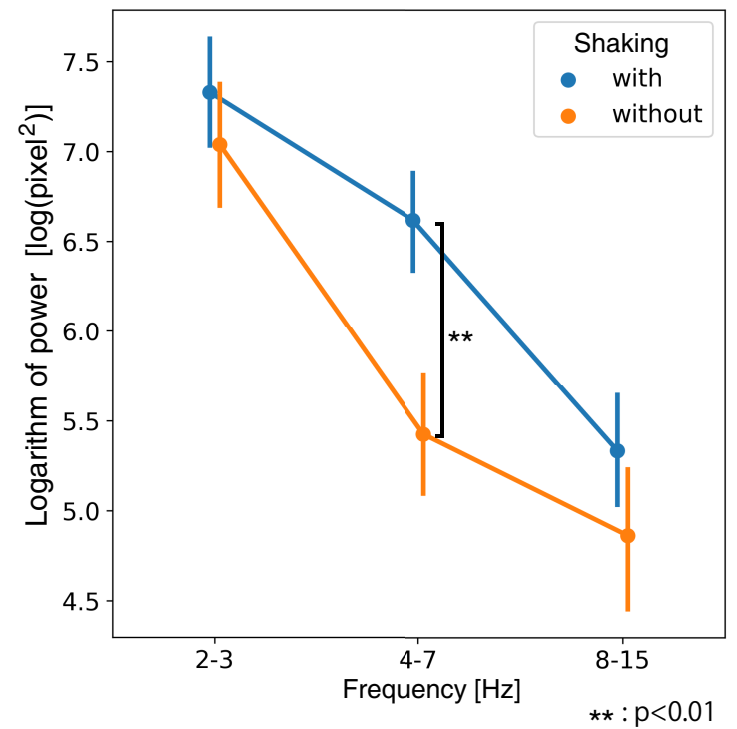

e

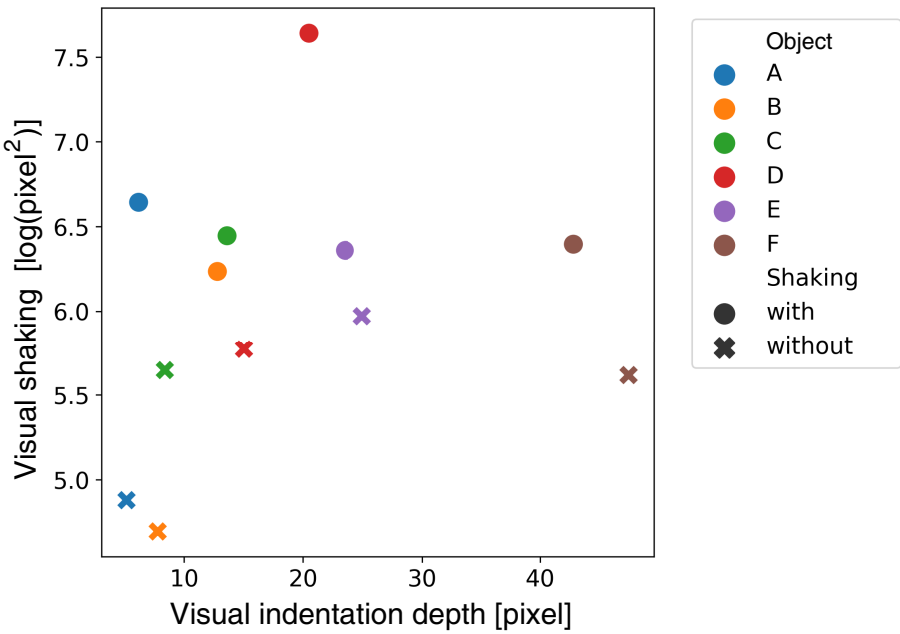

Figure 1. a, Appearance of objects. b, Force-displacement curves of objects. c, Applied max force for each condition in video clips. d, Logarithm of power of optical flow vectors (Error bars denote 95\%CI). e, Visual parameters of each video clip. 


\section{Results}

\section{Stimuli analysis}

The purpose of this study was to investigate how observers visually estimated the force applied on a target object by an actor. For this purpose, we controlled two types of visual information: the first corresponded to the induced tremor and the second corresponded to the change in the object' $s$ appearance in the video clips shown to the observers.

In this study, instead of causing the genuine induced tremor in an actor's body, the actor imitated the induced tremor by shaking their index finger as well as some parts of the hand. We call the imitated induced tremor as imitated shaking. The reason why we didn't use the genuine induced tremor was that we would like to keep the force that the actor applied to the object approximately constant in all video clips (Fig. 1c). By doing so, we tried to rule out the involvement of unintended factors (that is, other than the imitated shaking) in the estimation of force. For example, it is known that the color pattern in a fingertip can be a cue to the force direction ${ }^{12,13}$. By applying approximately constant forces to the object, we tried to remove the effect of undesired cues, such as the finger tip's color pattern, on the human observers' estimation of force . To quantify the shaking, we analyzed motion vectors at four finger-hand positions: the first finger joint, the second joint, the third joint and the fingertip. We conducted a fast Fourier transform on the temporal pattern of the motion vectors at each position and calculated the logarithm of power spectrum. As a result, a difference between the conditions with and without shaking was found only in a specific temporal frequency band (4-7 Hz) (Fig. 1d), which overlaps with temporal frequency bands in which the induced tremor is observed ${ }^{11}$.

Regardless of the presence/absence of finger shaking, the objects were actually pushed with a maximum force of about 1.5 - $2.0 \mathrm{~kg}$ (Fig.1c). While keeping the actor's force approximately constant, another issue can affect the experimental design. Specifically, when a similar level of force is applied to the objects whose force-displacement curves are the same, the change in the appearance of the object will be almost constant among the objects. To overcome this issue, we used objects that had different force-displacement curves so that the appearance change would be different when a similar level of force was applied (shown in Figs. 1a,b). As a result, different amounts of deformation (that is, indentation depth) could occur even when a similar level of force was applied to the object. As shown in Fig. 1c, the force actually applied to the object by the actor was almost constant regardless of the type of object or the presence of shaking. However, the amount of deformation, which was calculated by the motion vectors of the finger tip, varied with the objects (shown in the x-coordinate of markers in Fig. 1e), depending on their force-displacement curve.

Based on these analyses of the stimulus characteristics, we believe that it is appropriate to use video clips showing shaking and objects with different force-displacement curves as stimuli.

\section{Brief description of experiment procedure}

In this experiment, observers were asked to watch the clips in which an object was deformed by being pressed with the index finger of an actor's right hand, and to report how much force the actor applied to the object on a 100-point visual analog scale. In addition to the force estimation, we explored how estimation of the softness of the object varied with the parameters we wanted to check. Previous studies have shown that the amount of deformation of the object governs the perceived softness ${ }^{14,15}$. On the other hand, it is not clear how the visual parameters determining the force estimation could influence the estimation of softness. We explored how both imitated shaking and the amount of deformation of the object affected the apparent softness of the objects. We used in total 12 different videos, which were a combination of 6 object conditions with different force-displacement curves, each with 2 shaking conditions, namely, with or without imitated shaking.

\section{Force rating}

The force rating scores for each combination of object and shaking conditions are shown in Fig. 2a. First of all, in order to investigate whether the force rating scores for the 12 videos were dependent on the parameters that we set, a two-way permutation analysis of variance ${ }^{16,17}$ was conducted with the six object conditions and the two shaking conditions as withinobserver factors. The significance of $\mathrm{p}$ values was drawn from 999 permutations of the data. The significance level was then set at 0.05. The main effects of the shaking $\left[F=103.14, p<0.001, r^{2}=0.024\right]$ and of the object $[F=117.42, p<0.001$, $\left.r^{2}=0.137\right]$ were significant. The interaction was also significant $\left[F=4.42, p<0.001, r^{2}=0.005\right]$. See Supplementary Note $\mathrm{S} 1$ for post-hoc tests.

Next, a generalized linear model (GLM) was used to analyze the effect of parametric aspects of visual features in the stimuli on the force rating scores. As mentioned above, we hypothesized that the two types of visual information, the "visual shaking" (the logarithmic power of the amplitude spectrum of image motion vectors) and the "visual indentation depth" (the size of the indentation depth calculated by the motion vectors of the finger tip), would be effective for force estimation. Fig.1e shows how these visual parameters vary with the object used and the presence/absence of shaking. We fitted a model that regressed force rating with visual shaking and visual indentation depth as factors (see prediction lines in Fig. 2a). Since the force rating scores are a positive continuous value, the GLM employed a logarithmic link function with a gamma distribution. 


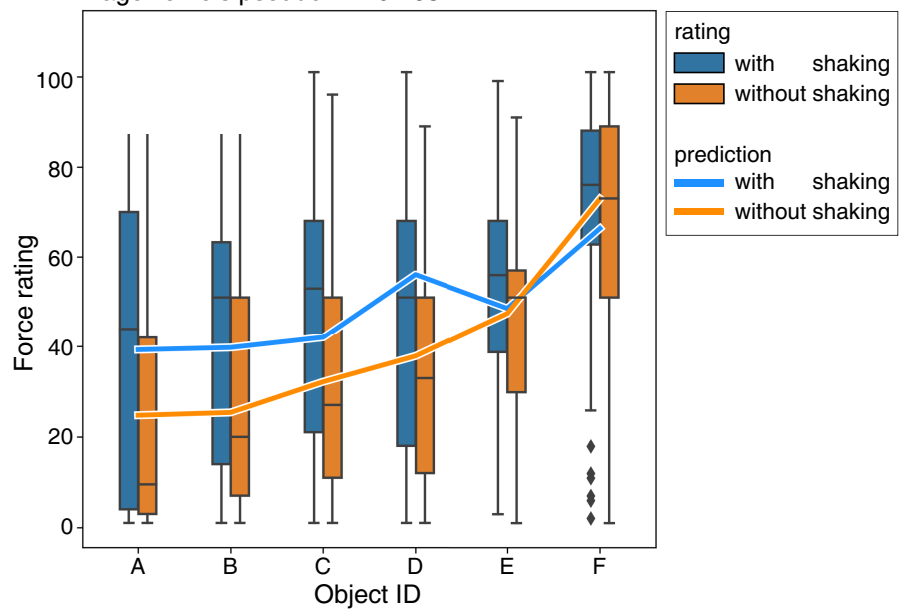

b
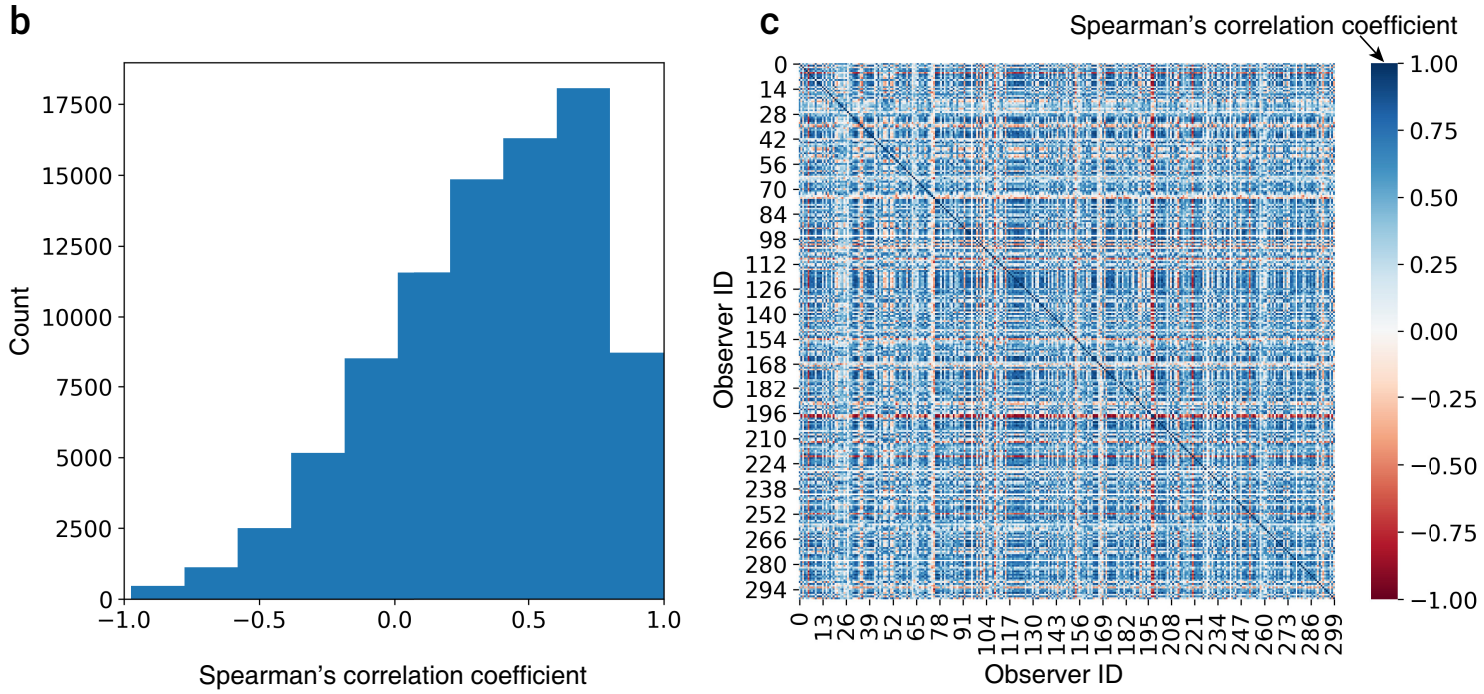

Figure 2. a, Force rating for each object with or without shaking. Predicted value of fitted GLM is also plotted. b, Histogram of Spearman's rank correlation coefficients of force rating between observers. c, Heatmap of the correlation coefficients.

As the result of a likelihood ratio test (Type II test), the main effect of visual shaking was significant $\left[d f=1, \chi^{2}=152.09\right.$, $p<0.001]$. Moreover, the main effect of the visual indentation depth was also significant $\left[d f=1, \chi^{2}=561.35, p<0.001\right]$. The interaction was also significant $\left[d f=1, \chi^{2}=14.78, p<0.001\right]$. The result suggests that human observers use the visual shaking, visual indentation depth, and their interaction to estimate the force that the actor applies to the object. To check which parameters contributed more to the regression, we compared the standardized partial regression coefficients among the factors. They were all significant $(p<0.05)$, and the standardized partial regression coefficient of the visual shaking was $0.120 \pm 0.012$, that of the visual indentation depth was $0.240 \pm 0.012$, and that of the interaction term was $-0.064 \pm 0.017$. This suggests that the visual indentation depth was more critical than the visual shaking. Regarding the goodness of fit of GLM, Nagelkerke's pseudo R-squared was 0.163 . As can be seen from the long boxes and whiskers in the box plot of Fig. 2a, the level of the force rating scores varied among the observers, and this might have resulted in the relatively low pseudo R-squared value. Figs. $2 \mathrm{~b}$ and $\mathrm{c}$ show the Spearman's rank correlation coefficient of force rating between observers. We calculated 10,000 bootstrap samples ${ }^{18}$ of the average value of the correlation coefficient and the bootstrap average was 0.343 (95\% CI(confidence interval): 0.342-0.346). Since the $95 \%$ CI did not overlap with zero, we conclude that the correlation coefficient was significantly larger than 0 while it was not necessarily high. Importantly, some pairs of observers showed negative correlation coefficients. The results indicate that the low pseudo R-squared value comes not from the large variance of the force rating scores along the GLM model, which would be consistent with our hypothesis, but from some systematic effect of the visual shaking and visual indentation depth, which is possibly different from our hypothesis. 
To explore whether the individual difference arose due to systematic effects of the visual shaking and visual indentation depth, we divided the observers into four groups on the basis of the sign (positive or negative) of the correlation coefficient between visual shaking and force rating as well as the sign (positive or negative) of the correlation coefficient between visual indentation depth and force rating. Of the 300 observers, omitting six observers who showed zero correlation coefficients, the 294 observers were divided into four groups. We expected that within each group the inter-observer correlation coefficients would be higher than those before the grouping. For each group, we calculated the correlation coefficient of the force rating scores between each pair of observers (see Fig. 3). There were no significant pairs of negative inter-observer correlation coefficients in any of the groups. In addition, the average value of the correlation coefficient between the observers in each group was 0.495 , which was larger than that in the case before grouping. The increase in the inter-observer correlation coefficient suggests that the within each group, the individual difference is attenuated in comparison with the population before grouping. 

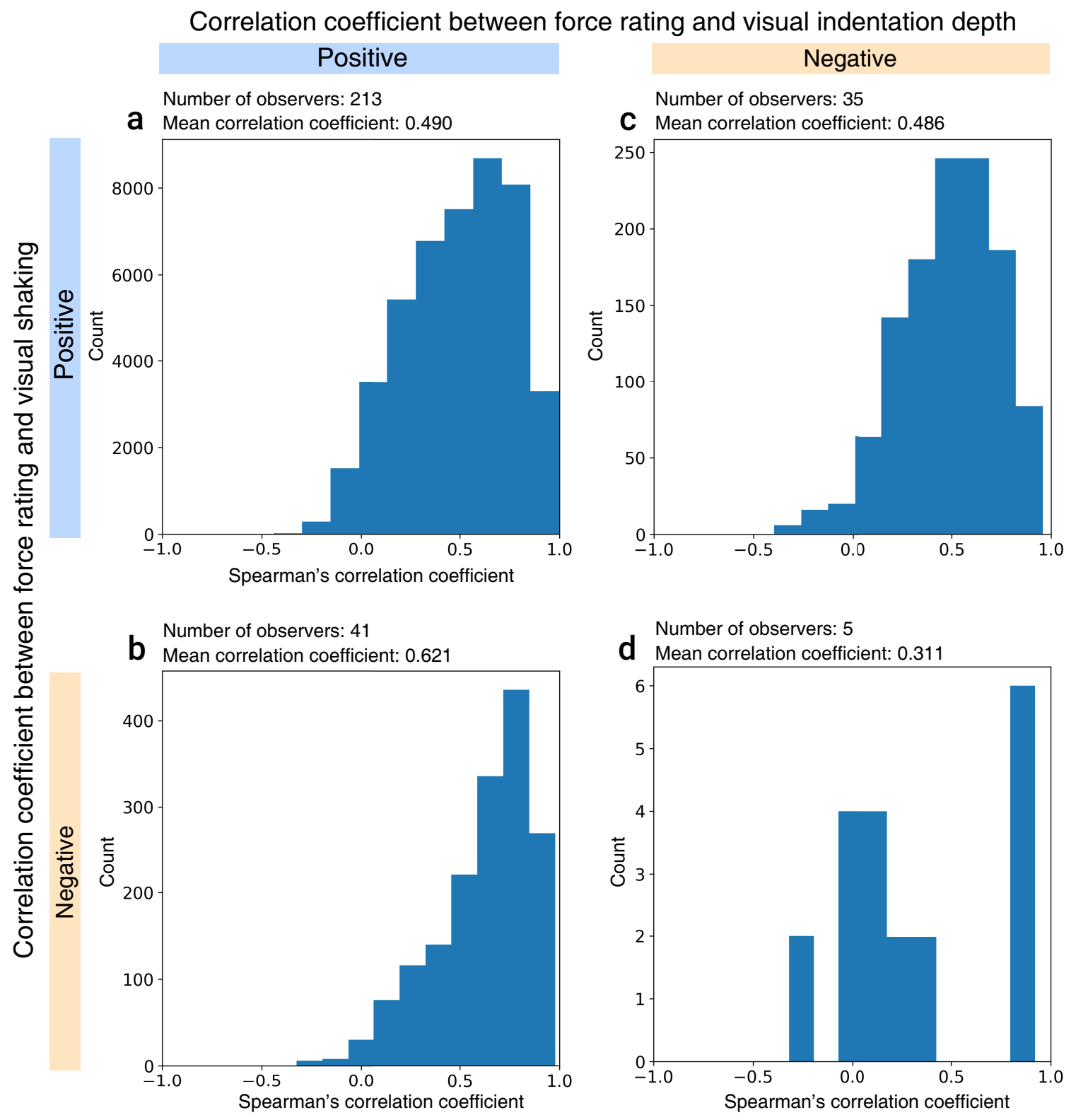

Number of observers: 5

d Mean correlation coefficient: 0.311
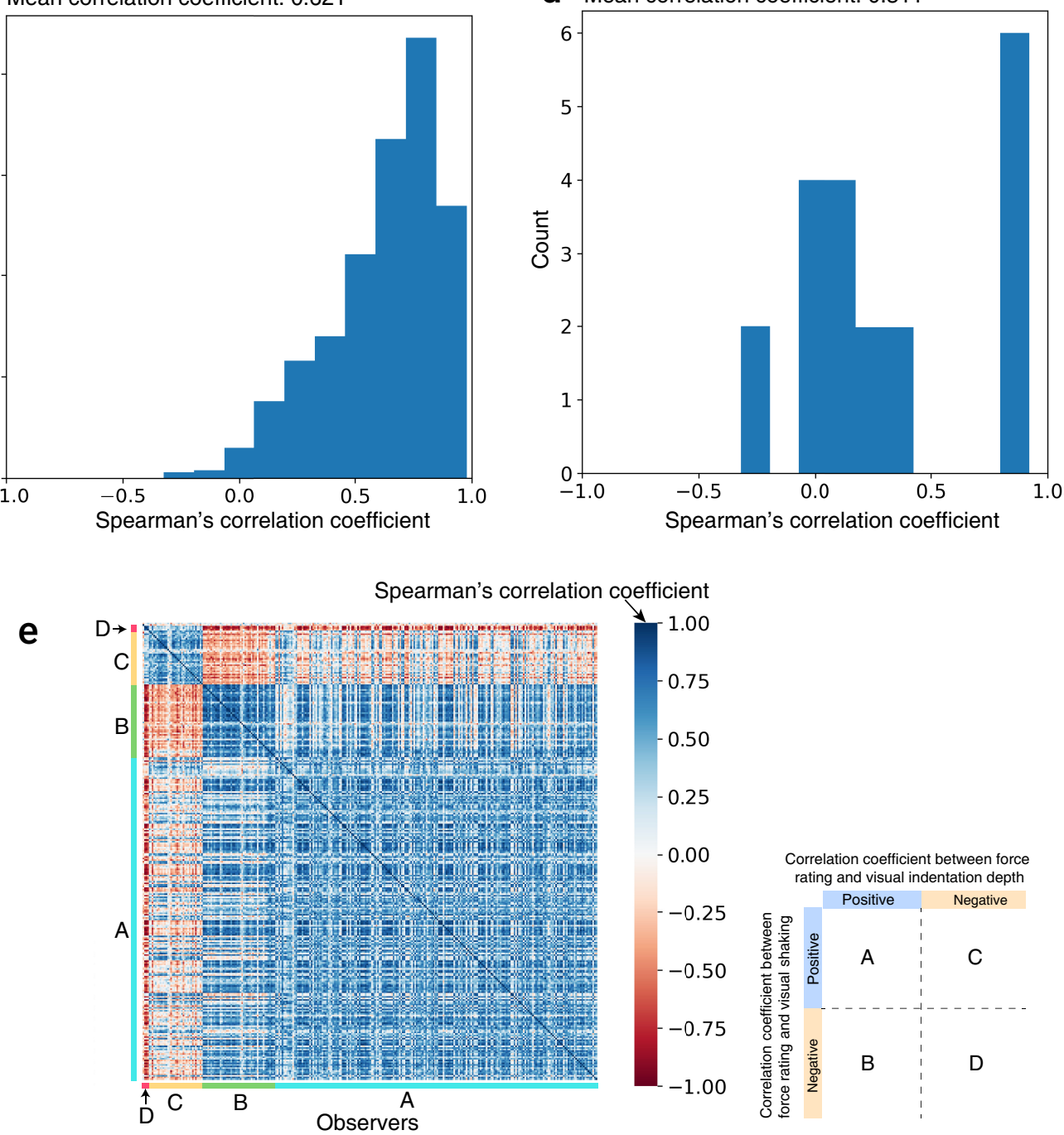

Figure 3. a-d, Histogram of Spearman's rank correlation coefficients of force rating between observers within each group. e, Heatmap of Spearman's rank correlation coefficients of force rating between observers, after sorting the observers into groy/AS 4 
Next, we tried to examine whether the way the observers used the visual information (visual shaking and visual indentation depth) was actually different among the groups. Fig. 4 shows the group-wise box-plot of the observer's force rating scores and the prediction of the scores on the basis of GLM. The average value of Nagelkerke's pseudo R-squared among the four groups was 0.243 , which was higher than the values before grouping. As can be seen from the pattern of the GLM prediction, the way the observers used visual information in the force rating tasks was different depending on the group. As a control analysis to evaluate the goodness of fit, we randomly divided the observers into four groups and conducted the GLMs for each group. As a result, $95 \% \mathrm{CI}$ of averaged pseudo R-squared of the control analysis ranged from 0.163 to 0.170 . The pseudo R-squared in this control analysis was smaller than the one in the former analysis where we grouped the observers on the basis of the correlation coefficients between the force rating score and the visual shaking/visual indentation depth. The results indicate that the individual difference occurred because some observers interpreted the visual shaking and visual indentation depth in a different way from our hypothesis. On the other hand, it should be noted that the largest number of the observers were classified into the group that was consistent with our hypothesis, in which the correlation coefficient between visual shaking and force rating was positive and the correlation coefficient between visual indentation depth and force rating was also positive.

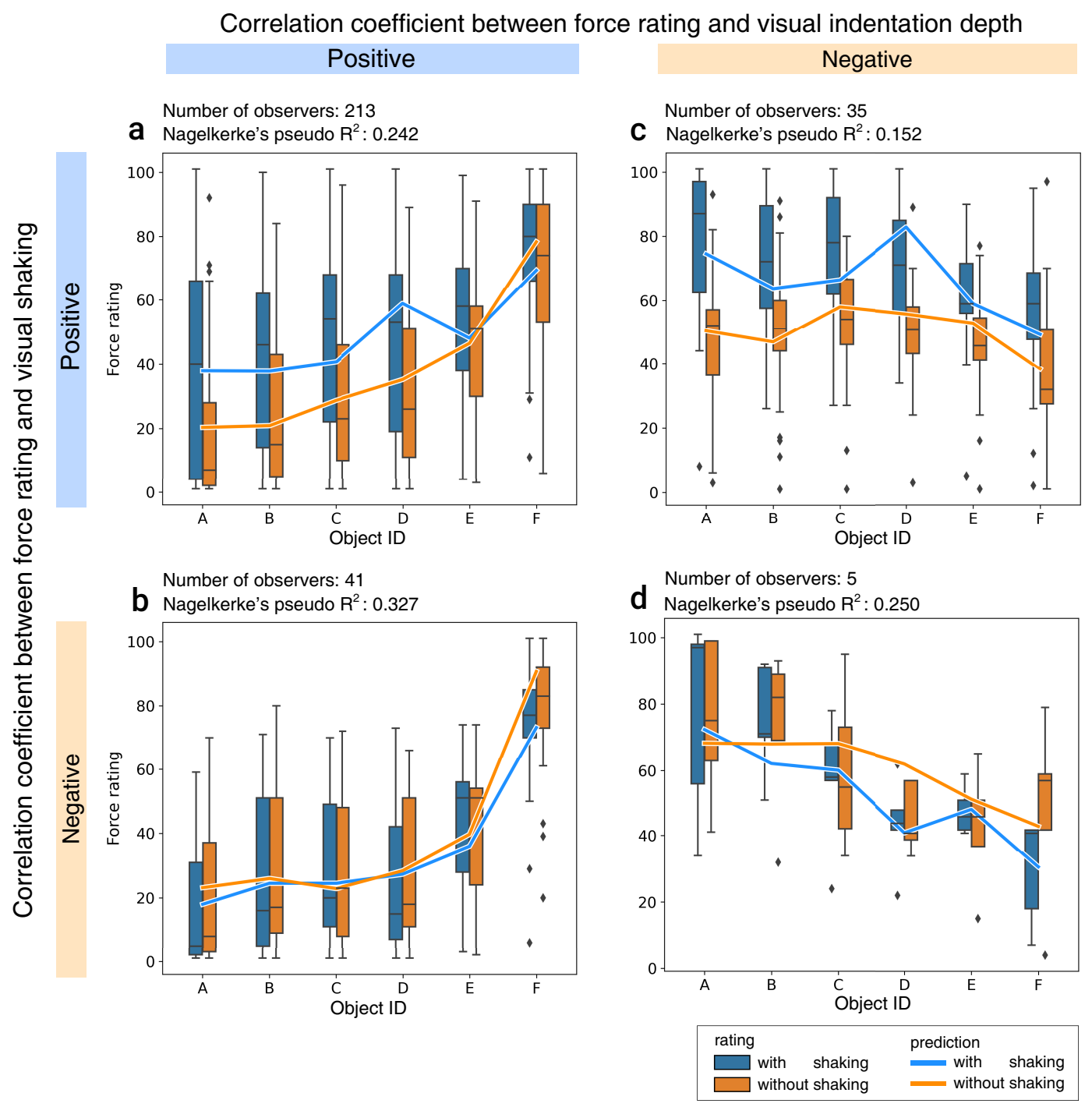

Figure 4. a-d, Force rating for each object, with or without shaking, for each group. Predicted value of group-wise fitted GLM is also plotted.

\section{Softness rating}

The softness rating scores for each combination of object and shaking condition are shown in Fig. 5a. In order to investigate whether the softness rating scores evaluated by the observers varied with the type of object in the video clips and the presence or 
absence of the imitated shaking, a two-way permutation analysis of variance was again conducted with the six object conditions and two shaking conditions as within-observer factors. The main effect of the object was significant $[F=538.05, p<0.001$, $\left.r^{2}=0.426\right]$. On the other hand, the main effect of shaking was not significant $\left[F=0.73, p=0.473, r^{2}=0.0001\right]$. Interaction between the two factors was significant $\left[F=4.31, p<0.001, r^{2}=0.003\right]$. See Supplementary Note 2 for post-hoc tests.
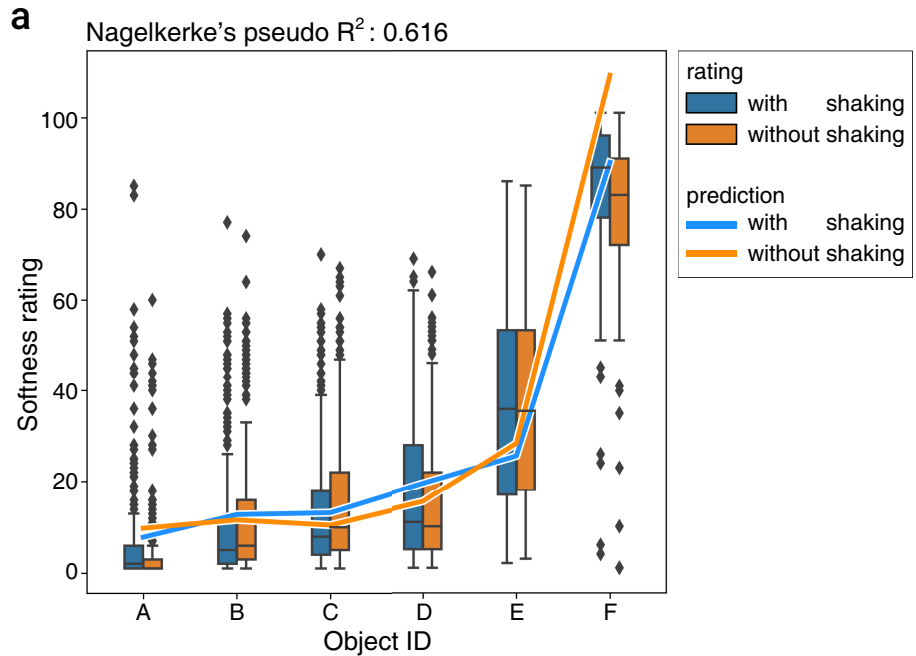

b
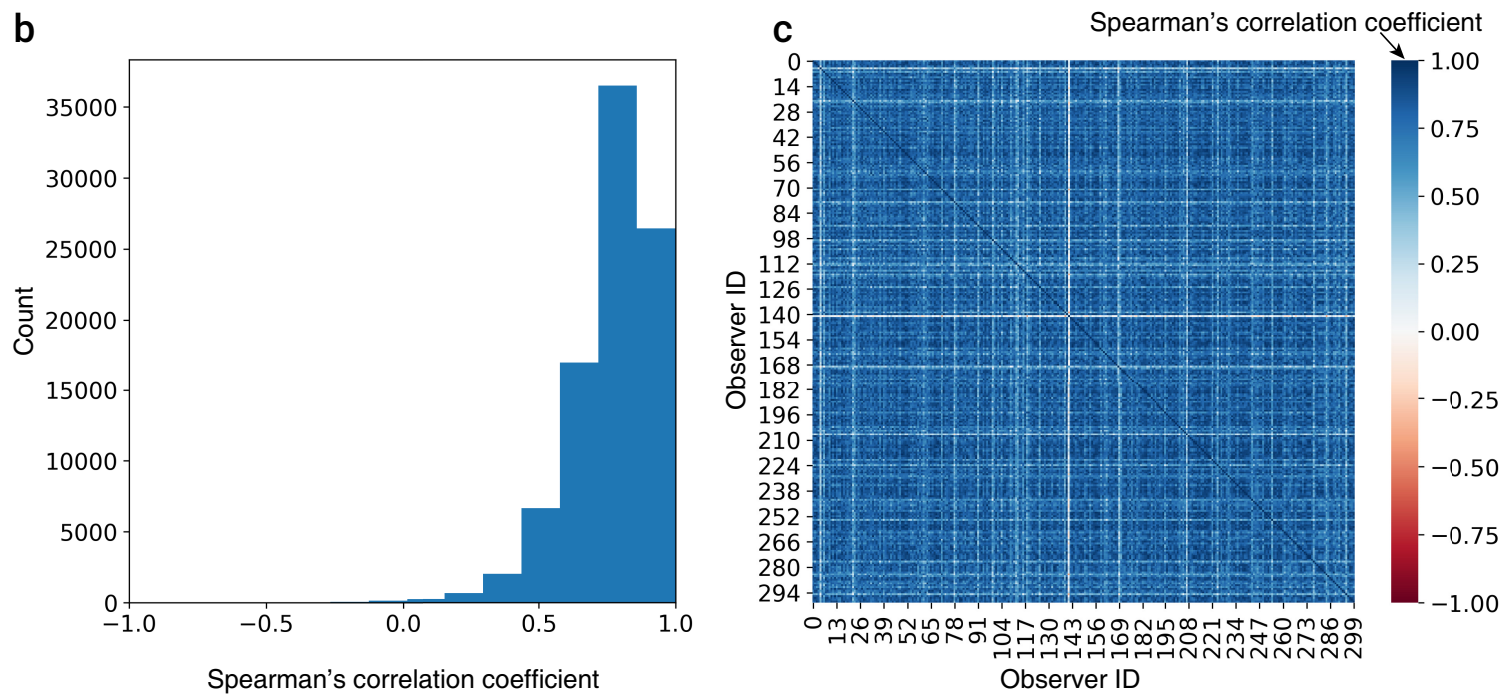

Figure 5. a, Softness rating for each object, with or without shaking. Predicted value of fitted GLM is also plotted. b, Histogram of Spearman's rank correlation coefficients of softness rating between observers. c, Heatmap of correlation coefficients.

Next, as in the case of the force rating scores, we investigated whether visual shaking and the visual indentation depth could explain the variation in the softness rating scores. We fitted a GLM that regressed the softness rating scores with visual shaking and visual indentation depth as factors (see Fig. 5a). Since the softness rating scores are also a positive continuous value, it was again modeled by a logarithmic link function with a gamma distribution. As a result of a likelihood ratio test (Type II test), the main effect of the visual shaking was significant $\left[d f=1, \chi^{2}=14.36, p<0.001\right]$. The main effect of the visual indentation depth was also significant $\left[d f=1, \chi^{2}=2426.25, p<0.001\right]$. Interaction between the two factors was also significant $\left[d f=1, \chi^{2}=6.54, p<0.011\right]$. Comparing the standardized partial regression coefficients among the factors, we observed that the coefficients were all significant $(p<0.05)$. The standardized partial regression coefficient of the visual shaking was $-0.049 \pm 0.019$, that of the visual indentation depth was $0.834 \pm 0.018$, and that of the interaction term was $0.074 \pm 0.026$. This showed that the visual shaking had a significant negative effect and the visual indentation depth had a significant positive effect on the softness rating scores. Comparing the absolute value of the standardized partial regression coefficients of both parameters, we found that the visual shaking had much less effect than the visual indentation depth. The goodness of Nagelkerke's pseudo R-squared for the GLM fitting was 0.62, which was significantly larger than that for the force 
rating scores. In addition, calculating the Spearman's rank correlation coefficient of the softness rating between each two of the observers Figs. $5 \mathrm{~b}$ and c, we found that the mean rank correlation coefficient between each pair of the observers was 0.763 (95\%CI: 0.762-0.764). This value was also larger than the rank correlation coefficient for force rating. Given the high pseudo R-squared and the inter-observer correlation coefficient, we concluded that the visual information used in the softness rating task and its interpretation by the observers was consistent among the observers.

\section{Discussion}

The purpose of the present study was to examine how human observers estimate another person's force from visual information only. We found that the other person' s force was estimated to be larger with a larger magnitude of visual shaking in the actor's movement of finger and hand. The results add new evidence to the literature showing that human observers can visually read the kinematics of other people' s motor action ${ }^{5-8}$. Moreover, consistent with our hypothesis, force applied by another person was estimated to be larger with a larger visual indentation depth. To sum up, the results newly suggest that human observers infer the force another person applies by looking at the variations of both an actor's bodily movement and the consequence of the action in the external world.

The induced tremor is a kind of involuntary muscle contraction that occurs under appropriate loading ${ }^{11}$. Why can human observers use the visual correlation of such involuntary motor components to judge the force being applied by another person? One possibility is that the observers have possibly experienced an induced tremor when they apply a strong force to an object (for example, when they try to open the tough screw lid of a bottle) and the past experience of the induced tremor can influence the visual interpretation of another person's induced tremor through a mechanism like perceptual resonance ${ }^{19}$. This sort of visual understanding of other's force may contribute to the promotion of communication with another person through the combination of visual observation and motor action ${ }^{20}$.

The visual indentation depth influenced both the force and softness rating scores (Fig. 2a). A larger value of the visual indentation depth generally caused a higher rating score of the force applied by another person. Consistent with the previous studies ${ }^{14,15}$, in another session using a different set of the observers, the softness rating scores also increased with the visual indentation depth. Taken together, the observers reported a stronger force being applied by the actor in the video under conditions in which the object was seen as being softer. This is apparently odd because in general, making an indentation in softer objects should have required a smaller magnitude of force than in harder objects. One possible idea to explain this oddness is that the brain estimates another person's force separately from the object softness. There are two possible causes for the large magnitude of indentation in an object. One cause might be a stronger force applied to the object while the other cause might be the softer property of the object. The result suggests that the brain assumes the former cause when estimating the force applied by another person in our experimental setting. On the other hand, the outcome of the force estimation does not seem to be employed when estimating the object' $\mathrm{s}$ softness. This interpretation can be supported by our permutation analysis of variance showing that the factor of visual shaking did not influence the softness rating task. Moreover, the analysis with the GLM showed the contribution of the visual shaking to the softness rating was very small. The results indicate that the brain estimates the apparent softness of an object to some extent separately from the estimation of the force applied by another person.

Why was the softness perception not influenced by visual shaking? Softness is the subjective impression of the physical compliance $^{21}$ that is computed by using the magnitudes of indentation and force. Hence, as shown in haptic softness perception $^{22}$, we expected that human observers would visually estimate softness based on both indentation depth and force. However, the softness estimation was not strongly influenced by visual shaking, which was a potential visual feature of the force being applied by another person. As described in the previous study ${ }^{22}$, in a difference from the tactile/haptic system, the visual system does not have any sensor to directly detect force. Thus, the brain needs to indirectly infer force on the basis of other visual features (like visual shaking in this study). On the other hand, the visual system is sensitive to position changes and motion, in other words, it can robustly detect the variation of indentation depth. If the apparent softness is determined by the optimal integration of several cues, the indentation depth which can be robustly detected is likely of greater significance than the force which can only be estimated indirectly from several visual cues. It has been shown that the haptic softness perception is influenced by visual indentation ${ }^{14}$, which also supports the idea that the indentation depth plays a robust role in the determination of object' s perceived softness. The previous notion can well explain the data that the softness estimation is mainly determined by the visual indentation depth.

The population in Fig. 4a likely interprets the visual shaking and visual indentation depth consistently with our hypothesis, but some populations of the observers perhaps exploited different interpretations of visual parameters to determine the force applied by another person (shown in Figs 4b, c, and d). Figs. $4 \mathrm{~b}$ and d show the data of the populations reporting the higher force rating scores with the smaller visual shaking. It is unclear why the observers in the population reported in this way, but one of the speculations might be that the observers interpreted visual shaking as coming from some pathological tremor ${ }^{23}$ and hence the visual shaking did not stem from the application of strong force to a object. In line with this unusual interpretation, 
the visual shaking might be interpreted as evidence of the weak power of an actor. Figs. $4 \mathrm{c}$ and d show the data of the populations reporting the higher force rating scores with the smaller visual indentation depth (the object A showed a smaller visual indentation depth than the object F, as shown in Fig. 1e). The observers in those populations likely considered that the variation in the visual indentation depth stemmed from the variation in the object' $\mathrm{s}$ softness, and also that the actor applied a smaller magnitude of force to make an indentation in the softer object. Although there are systematic tendencies for some populations of the observers to use interpretations different from our expectation, the majority of the observers reported the force rating scores in a way consistent with our hypothesis. In future research, we need to understand why such differences in the interpretation of visual shaking and visual indentation depth occur in some populations of observers.

In this study, as stimuli, we used video clips in which the top surface of an elastic object was pushed with the index finger of an actor, and found that the visual shaking is one of several effective cues to the estimation of the force being applied. Besides the situation we experimentally investigated, in everyday life there are many scenarios in which another person applies force to an external object. For example, it is known that human observers can visually estimate the weight of a box that is lifted by another person ${ }^{24}$. It is an intriguing issue whether the estimate of weight and the lifter's force can be influenced by the visual shaking in the arm of the person lifting the box. Moreover, it is also an important issue whether the estimation of force that another person applies is influenced by characteristics of the person who applies force to the object. For example, future studies need to examine how the age and gender which are inferred from the shape and size of an actor's hand determine the visual estimation of force that the actor applies. Finally, it is common to observe that another person pushes or manipulates external objects by using tools such as knives, forks, chopsticks, scissors, baseball bats, shovels, etc. It is an interesting issue whether the visual shaking of these tools also plays a critical cue to the estimation of the force being applied.

\section{Methods}

\section{Observers}

In total, 600 people participated in the experiment. The participants, with a mean age of 35.35 (SD: 8.51), consisted of three age groups $(20 \mathrm{~s}, 30 \mathrm{~s}$, and $40 \mathrm{~s})$ each made up of 100 men and 100 women. The 600 participants were classified into two groups each having 300 participants so that age and gender were balanced between the groups. Observers in one group performed the force rating task while those in the other group performed the softness rating task. They were recruited online by a crowdsourcing research agent in Japan and were paid for their participation. Only people who could participate in the experiment using their own personal computer were recruited. They were unaware of the specific purpose of the experiment. Ethical approval for this study was obtained from the ethics committee at Nippon Telegraph and Telephone Corporation (Approval number: R02-009 by NTT Communication Science Laboratories Ethics Committee). The experiments were conducted according to the principles that have their origin in the Helsinki Declaration. Written informed consent was obtained from all observers in this study.

\section{Stimuli}

The stimuli were video clips that showed an elastic cube of which the top surface was pushed down by an actor's index finger of the right hand. The video resolution was $288 \times 512$ pixels at 29.97 frames per second.

\section{D Printed Object}

We attempted to replicate six cubic metamaterials introduced $\mathrm{in}^{25}$ by using the identical types of material (TangoBlackPlus) and 3D printer (Stratasys Obje500). Fig. 1a,b show the photograph of the objects and their force-displacement curves respectively. The length of each edge of the cube was $42 \mathrm{~mm}$. Each cube contained 169 cylindrical holes. There were two sizes of hole for each object, which were different depending on the object as shown in Supplementary Table 1. The distance between the holes was $3.0 \mathrm{~mm}$. These configurations were identical to those used in the previous study ${ }^{25}$. Because the cube's surfaces in the previous study had a striped pattern and thus were not smooth, we made the surfaces flat because we wanted to remove the effect of a striped pattern on the perception of softness.

To characterize the material property, we performed uniaxial load testing. An increasing force was applied to the cubes from the top at to give a displacement at a constant speed of $1 \mathrm{~mm} / 6$ seconds, and the corresponding force was recorded using a force tester (MCT-2150, A\&D Co., Ltd.). Fig. 1b shows the measured force-displacement curves. Although there was a difference in the force-displacement curve from the study ${ }^{25}$, this could be attributed to the aforementioned absence of surface striped patterns.

\section{Video Clip}

We set the orientation of the object so that the surface with cylindrical holes was directed toward the actor. We took videos from diagonally above so that the top surface pushed by the finger could be seen. The viewpoint of the camera was $30 \mathrm{~cm}$ in the front direction of the object, $15 \mathrm{~cm}$ to the side, and $20 \mathrm{~cm}$ above. The camera lens was oriented towards the object. We filmed the actor's right index finger pressing on the top of the object to deform it, and then relaxing his finger to return the object to 
its original shape. The recorded scene in the video started from the time when the finger was stationary and in contact with the top surface of the object. The actor practiced in advance so that it would take approximately 3-4 seconds to complete one cycle of the pushing-relaxing movement and the maximum pressing force would reach $1.5-2.0 \mathrm{~kg}$. (see the actual max force applied for each object in Fig. 1c. We took videos for each object under the following two shaking conditions: one condition (i.e., without-shaking condition) in which the actor pushed naturally and the other condition (i.e., with-shaking condition) in which the actor shook his finger and hand while pushing. Between the two conditions, the main difference in visual signals was the presence of visual shaking due to the imitation of an induced tremor. Thus, there were in total 12 conditions (6 object conditions $\times 2$ shaking conditions). See Supplementary Videos 1-12 corresponding to the 12 conditions and Supplementary Figure 1 that shows snapshots of the stimulus videos without shaking.

\section{Quantifying visual features}

To quantify the image information relevant to the indentation depth and the shaking, we computed optical flows using the Lucas-Kanade algorithm ${ }^{26}$ at the four image positions including the 1st joint, the 2nd joint, the 3rd joint, and the fingertip of the index finger of the actor (shown in Supplementary Figure 2). Regarding the indentation depth, the maximum amount of the vertical displacement from the initial position of the fingertip was calculated as the visual indentation depth. The calculated visual indentation depth for each video clip is shown as the x-coordinate of each marker in Fig. 1e. Regarding the visual shaking, we conducted a fast Fourier transform for the temporal variations of the optical flow vectors along both horizontal and vertical dimensions at the four positions and obtained the logarithmic powers of amplitude spectra, which were summed across the horizontal and vertical dimensions as well as within each octave band $(2-3 \mathrm{~Hz}, 4-7 \mathrm{~Hz}$, and 8-15 Hz). To determine which octave band characterized the shaking, we conducted an Aligned Rank Transform (ART) ${ }^{27}$ on the data and then conducted a two-way ANOVA using factors of the octave band and the shaking condition on the aligned ranks. The main effect of the octave band was significant $[d f=2, F=286.430, p=0.001]$. The main effect of the shaking condition was significant $[d f=1, F=9.570, p=0.003]$. The interaction effect was also significant $[d f=2, F=14.165, p=0.001]$. The post-hoc test revealed that there were no significant differences between with- and without-shaking for the octave bands of 2-3 $\mathrm{Hz}$ or 8-15 $\mathrm{Hz}(p>0.05)$, but that there was a significant difference between with- and without- shaking for the octave band of 4-7 Hz $(p<0.001)$. Therefore, in this study, we used the logarithmic powers of the amplitude spectra of the optical flow vectors for the octave band of 4-7 Hz as the feature of visual shaking for further analysis. The logarithmic power of the amplitude spectra for each object is shown as the y coordinate of each marker in Fig. 1e

\section{Procedure}

The experiment was programmed using jsPsych ${ }^{28}$. Each observer participated in the experiment in his or her own environment using a personal computer. Neither observation distances nor screen sizes were controlled. Although the presentation accuracy was not measured, it has been reported that stimulus timing control with jsPsych is sufficient to conduct online psychological experiments ${ }^{29}$.

After viewing each video, observers estimated the softness or the applied force and responded using a visual analog scale $(\mathrm{VAS})^{30}$ ranging from 0 to 100 with 100 scale divisions. In the case of estimating force, the "No force at all" anchor was placed on the left side of the scale and the "Largest force that you can imagine" anchor was placed on the right side of the scale. In the case of answering softness, the "Not soft at all" anchor was placed on the left side of the scale and the "Softer than anything you can imagine" anchor was placed on the right side of the scale. The experiment was composed of initial familiarization and then the actual test phases. In the familiarization phase, each observer was asked to provide answers for all 12 conditions. After these were completed, the test phase started, in which each observer provided an answer for each of the 12 conditions. The presentation order of the 12 conditions was assigned pseudo-randomly to each observer.

\section{Data Analysis}

In order to investigate whether the rating scores for force or softness for the 12 videos differed depending on the type of object shown in the video and the presence or absence of shaking, a two-way permutation analysis of variance ${ }^{16,17}$ was performed.

Next, a GLM was used to analyze the effect of visual indentation depth and visual shaking. Since the force rating is a positive continuous value, it was modeled by a logarithmic link function with a gamma distribution. To determine which factor is significant, a likelihood ratio test (Type II test) was performed. To determine which factor is more significant, standardized partial regression coefficients were compared. Nagelkerke's pseudo R-squared was calculated to check how the GLM could explain our results. To quantify the individual differences, we calculated the inter-observer correlation coefficients (the Spearman's rank correlation coefficients) for rating scores between each two of the observers.

\section{References}

1. Aglioti, S. M., Cesari, P., Romani, M. \& Urgesi, C. Action anticipation and motor resonance in elite basketball players. Nat. Neurosci. 11, 1109-1116, DOI: 10.1038/nn.2182 (2008). 
2. Becchio, C., Koul, A., Ansuini, C., Bertone, C. \& Cavallo, A. Seeing mental states: An experimental strategy for measuring the observability of other minds. Phys. Life Rev. 24, 67-80, DOI: 10.1016/j.plrev.2017.10.002 (2018).

3. Iacoboni, M. et al. Grasping the intentions of others with one' s own mirror neuron system. PLoS Biol. 3, e79, DOI: 10.1371/journal.pbio.0030079 (2005).

4. Kilner, J. M. More than one pathway to action understanding. Trends Cogn. Sci. 15, 352-357, DOI: 10.1016/j.tics.2011.06. 005 (2011).

5. Ansuini, C. et al. Grasping others' movements: Rapid discrimination of object size from observed hand movements. $J$. Exp. Psychol. Hum. Percept. Perform. 42, 918-929, DOI: 10.1037/xhp0000169 (2016).

6. Ansuini, C. et al. Predicting object size from hand kinematics: A temporal perspective. PLOS ONE 10, e0120432, DOI: 10.1371/journal.pone.0120432 (2015).

7. Hamilton, A. F. d. C., Joyce, D. W., Flanagan, J. R., Frith, C. D. \& Wolpert, D. M. Kinematic cues in perceptual weight judgement and their origins in box lifting. Psychol. Res. 71, 13-21, DOI: 10.1007/s00426-005-0032-4 (2007).

8. Ansuini, C. et al. Are we real when we fake? attunement to object weight in natural and pantomimed grasping movements. Front. Hum. Neurosci. 10, 471, DOI: 10.3389/fnhum.2016.00471 (2016).

9. Kilner, J. M., Vargas, C., Duval, S., Blakemore, S.-J. \& Sirigu, A. Motor activation prior to observation of a predicted movement. Nat. Neurosci. 7, 1299-1301, DOI: 10.1038/nn1355 (2004).

10. Stefani, E. D. et al. Learning new sport actions: Pilot study to investigate the imitative and the verbal instructive teaching methods in motor education. PLOS ONE 15, e0237697, DOI: 10.1371/journal.pone.0237697 (2020).

11. Durbaba, R., Cassidy, A., Budini, F. \& Macaluso, A. The effects of isometric resistance training on stretch reflex induced tremor in the knee extensor muscles. J. Appl. Physiol. 114, 1647-1656, DOI: 10.1152/japplphysiol.00917.2011 (2013).

12. Sun, Y., Hollerbach, J. M. \& Mascaro, S. A. Imaging the finger force direction. 2007 IEEE Conf. on Comput. Vis. Pattern Recognit. 1-6, DOI: 10.1109/cvpr.2007.383256 (2007).

13. Sugita, N., Iwai, D. \& Sato, K. Touch sensing by image analysis of fingernail. 2008 SICE Annu. Conf. 1520-1525, DOI: 10.1109/sice.2008.4654901 (2008).

14. Fakhoury, E., Culmer, P. R. \& Henson, B. The effect of indentation force and displacement on visual perception of compliance. In 2015 IEEE World Haptics Conference (WHC), 88-93 (IEEE, 2015).

15. Paulun, V. C., Schmidt, F., van Assen, J. J. R. \& Fleming, R. W. Shape, motion, and optical cues to stiffness of elastic objects. J. vision 17, 1-22 (2017).

16. Anderson, M. J. A new method for non-parametric multivariate analysis of variance. Austral ecology 26, $32-46$ (2001).

17. Anderson, M. J. \& Walsh, D. C. Permanova, anosim, and the mantel test in the face of heterogeneous dispersions: what null hypothesis are you testing? Ecol. monographs 83, 557-574 (2013).

18. Efron, B. \& Tibshirani, R. J. An introduction to the bootstrap (CRC press, 1994).

19. Schütz-Bosbach, S. \& Prinz, W. Perceptual resonance: action-induced modulation of perception. Trends Cogn. Sci. 11, 349-355, DOI: 10.1016/j.tics.2007.06.005 (2007).

20. Sebanz, N., Bekkering, H. \& Knoblich, G. Joint action: bodies and minds moving together. Trends Cogn. Sci. 10, 70-76, DOI: 10.1016/j.tics.2005.12.009 (2006).

21. Di Luca, M. Multisensory Softness (Springer, 2014).

22. Freyberger, F. K. B., Kuschel, M., Klatzky, R. L., Färber1, B. \& Buss, M. Visual-haptic perception of compliance: Direct matching of visual and haptic information. 2007 IEEE Int. Work. on Haptic, Audio Vis. Environ. Games 5-10, DOI: 10.1109/have.2007.4371577 (2007).

23. Elble, R. J. Tremor. In Neuro-Geriatrics, A Clinical Manual, 311-326, DOI: 10.1007/978-3-319-56484-5_20 (2017).

24. Runeson, S. \& Frykholm, G. Visual perception of lifted weight. J. Exp. Psychol. Hum. Percept. Perform. 7, 733-740, DOI: 10.1037/0096-1523.7.4.733 (1981).

25. Piovarči, M. et al. An interaction-aware, perceptual model for non-linear elastic objects. ACM Transactions on Graph. (TOG) 35, 1-13 (2016).

26. Lucas, B. D., Kanade, T. et al. An iterative image registration technique with an application to stereo vision (Vancouver, British Columbia, 1981). 
27. Wobbrock, J. O., Findlater, L., Gergle, D. \& Higgins, J. J. The aligned rank transform for nonparametric factorial analyses using only anova procedures. In Proceedings of the SIGCHI conference on human factors in computing systems, 143-146 (2011).

28. De Leeuw, J. R. jspsych: A javascript library for creating behavioral experiments in a web browser. Behav. research methods 47, 1-12 (2015).

29. Bridges, D., Pitiot, A., MacAskill, M. R. \& Peirce, J. W. The timing mega-study: comparing a range of experiment generators, both lab-based and online. PeerJ 8, e9414 (2020).

30. Crichton, N. Visual analogue scale (vas). J Clin Nurs 10, 706-6 (2001).

\section{Data availability}

The authors confirm that the data supporting the findings of this study are available within the article and its supplementary materials.

\section{Code availability}

The code used for the analysis in this study is available from the corresponding authors upon request.

\section{Author contributions statement}

Y.U. and T.K conceived and conducted the experiment, Y.U. and T.K analysed the results. All authors reviewed the manuscript.

\section{Additional information}

Competing interests Y.U and T.K are employees of NTT Communication Science Laboratories, which is a basic-science research section of Nippon Telegraph and Telecommunication corporation (NTT). There is a pending patent involving the reported research. There are no products in development or marketed products to declare. The pending patent does not alter the author's adherence to policies of Scientific Reports. 